\title{
In-situ formation of electron acceptor to inhibit charge separation of photo- electrochemical sensor of dopamine-based CdS/Au/GQDs
}

\begin{abstract}
A versatile photo-electrochemical (PEC) sensor protocol was established to quantitatively monitor dopamine (DA) levels by utilizing a triple interconnected structure of cadmium sulfide (CdS) modified with gold and graphene quantum dots (Au/GQDs). The introduction of $\mathrm{Au}$ and GQDs on the photocatalytic active center of $\mathrm{CdS}$ act as a charge separation mediator and photosensitizer, respectively, which are favorable for charge separation and transportation and PEC conversion. When the CdS/Au/GQDs photoelectrode was utilized for DA sensing in a weak alkaline solution, DA was oxidized and converted to poly(dopamine) (PDA), which possesses abundant benzoquinone (BQ) groups that act as electron acceptors. Consequently, the electron acceptors formed in-situ on the surface of the photoelectrode, reducing the anodic photocurrent signal. Under the optimal conditions, the photocurrent decreased when the DA concentration increased in a dynamic working range from 0.1 to $350 \mu \mathrm{M}$ and with a limit of detection (LoD) of $0.0078 \mu \mathrm{M}$. Herein, the proposed strategy involving photoelectron transfer between the electron acceptor and semiconductor provides a new and versatile protocol for PEC sensor development.
\end{abstract}

Keyword: Photo-electrochemical (PEC) sensor; Dopamine; CdS/Au/GQDs photoelectrode; Electron acceptor; Poly(dopamine) 